\title{
THE YONEDA ALGEBRA OF A GRADED ORE EXTENSION
}

\author{
CHRISTOPHER PHAN
}

\begin{abstract}
Let $A$ be a connected-graded algebra with trivial module $\mathbb{k}$, and let $B$ be a graded Ore extension of $A$. We relate the structure of the Yoneda algebra $\mathrm{E}(A):=\operatorname{Ext}_{A}(\mathbb{k}, \mathbb{k})$ to $\mathrm{E}(B)$. Cassidy and Shelton have shown that when $A$ satisfies their $\mathcal{K}_{2}$ property, $B$ will also be $\mathcal{K}_{2}$. We prove the converse of this result.
\end{abstract}

\section{INTRODUCTION}

Let $A$ be a connected-graded algebra over a field $\mathbb{k}$ generated in degree 1 , and let ${ }_{A} \mathbb{k}$ be the $A$-module $A / A_{+}$. We then have a bigraded algebra $\mathrm{E}(A):=\operatorname{Ext}_{A}(\mathbb{k}, \mathbb{k})$, called the Yoneda algebra of $A$. (Throughout, Ext and Tor refer to the functors on the graded category. We define $\mathrm{E}^{i}(A)=\bigoplus_{j} \mathrm{E}^{i, j}(A)$, where the index $i$ refers to the homological grading and $j$ refers to the grading inherited from $A$.) Frequently studied is a property introduced by Priddy in [9]: an algebra $A$ is Koszul if $\mathrm{E}(A)$ is generated in the first cohomological degree (equivalently, $\mathrm{E}^{i, j}(A)=0$ when $i \neq j$ ). For a comprehensive reference on Koszul algebras, see [8].

In [3] Cassidy and Shelton proposed the following generalization of Koszul:

Definition 1.1. A connected-graded algebra $A$ generated in degree 1 is a $\mathcal{K}_{2}$ algebra if $\mathrm{E}(A)$ is generated in the first two cohomological degrees.

This class includes the class of $\mathrm{N}$-Koszul algebras defined by Berger in [1]. For quadratic algebras, $\mathcal{K}_{2}$ is equivalent to Koszul. The study of $\mathcal{K}_{2}$ algebras is attractive because the first two cohomological degrees of $\mathrm{E}(A)$ are seen in a suitable presentation of $A$ : we have $\mathrm{E}^{1}(A) \simeq A_{1}^{*}$ while $\mathrm{E}^{2}(A)$ is roughly dual to the essential relations of $A$. The class of $\mathcal{K}_{2}$ algebras has been further studied in [2] and [7].

In addition to exploring the connections between $A$ and $\mathrm{E}(A)$, algebraists have investigated structural similarities between $\mathrm{E}(A)$ and $\mathrm{E}(B)$ when $B$ is an algebra related to $A$. For example (see [3, 8]), both the classes of Koszul and $\mathcal{K}_{2}$ algebras are closed under regular central extensions, Zhang twists, and tensor products.

Furthermore, both the class of Koszul algebras and the class of $\mathcal{K}_{2}$ algebras are closed under graded Ore extensions. Suppose $\sigma: A \rightarrow A$ is a graded algebra automorphism and $\delta: A(-1) \rightarrow A$ is a graded $\sigma$-derivation. Then the associated Ore extension $B:=A[z ; \sigma, \delta]$ is also a connected-graded algebra. (A primer on Ore extensions can be found in [5].) In [3], it was proved that if $A$ is a $\mathcal{K}_{2}$ algebra, then $B$ is also $\mathcal{K}_{2}$. This is a generalization of the wellknown result that such a graded Ore extension of a Koszul algebra is again Koszul.

Our goal in this article is to provide another connection between $\mathrm{E}(A)$ and $\mathrm{E}(B)$ when $B$ is such a graded Ore extension of $A$ :

Theorem 1.2. $B$ is $\mathcal{K}_{2}$ only if $A$ is $\mathcal{K}_{2}$.

Note that $B$ is quadratic if and only if $A$ is quadratic. We therefore obtain this corollary:

2000 Mathematics Subject Classification. 16E30, 16S36, 16S37, 16W50.

Key words and phrases. $\mathcal{K}_{2}$ algebra, Ore extension, Yoneda algebra. 
Corollary 1.3. B is Koszul only if $A$ is Koszul.

In Section 2 of this article, we describe a procedure that produces a projective resolution of ${ }_{B} \mathbb{k}$ based on a minimal projective resolution of ${ }_{A} \mathbb{k}$. In Section 3, we create some maps that relate the structure of $\mathrm{E}(A)$ and $\mathrm{E}(B)$. In Section 4, we prove Theorem 1.2. We conclude in Section 5 with some examples.

The author wishes to thank Kenneth Brown, Thomas Cassidy, Ulrich Krähmer, and Brad Shelton for their helpful comments and suggestions.

\section{PROJECTIVE RESOLUTION FOR ${ }_{B} \mathbb{K}$}

A projective resolution

$$
\cdots \rightarrow P_{n} \rightarrow P_{n-1} \rightarrow \cdots \rightarrow P_{0}
$$

of the $A$-module $M$ is minimal if the differentials in the sequence

$$
\operatorname{Hom}\left(P_{0}, \mathbb{k}\right) \rightarrow \cdots \rightarrow \operatorname{Hom}_{A}\left(P_{n-1}, \mathbb{k}\right) \rightarrow \operatorname{Hom}_{A}\left(P_{n}, \mathbb{k}\right) \rightarrow \cdots
$$

are all zero. In this section, we describe a procedure for constructing a projective resolution of the ${ }_{B} \mathbb{k}$ based on a minimal projective resolution of ${ }_{A} \mathbb{k}$. However, the resulting resolution for ${ }_{B} \mathbb{k}$ will not necessarily be minimal.

We begin by setting some notation in effect for the rest of the article. Let $V$ be a finitedimensional vector space over a field $\mathbb{k}$, and let $\mathbb{T}(V)$ denote the tensor algebra. Let $I \subseteq$ $\sum_{n>2} V^{\otimes n}$ be a finitely-generated homogeneous ideal of $\mathbb{T}(V)$ and let $A:=\mathbb{T}(V) / I$, which is a connected-graded $\mathbb{k}$-algebra generated in degree 1 . Let $\sigma: A \rightarrow A$ be a graded algebra automorphism, and $\delta: A(-1) \rightarrow A$ be a graded $\sigma$-derivation-that is, for $a_{1}, a_{2} \in A$, we have

$$
\delta\left(a_{1} a_{2}\right)=\delta\left(a_{1}\right) \sigma\left(a_{2}\right)+a_{1} \delta\left(a_{2}\right) .
$$

Let $B:=A[z ; \sigma, \delta]$ be the associated Ore extension-that is, $B=\bigoplus_{n \geq 0} z^{n} A$ as an $A$-module, and for $a \in A$,

$$
a z=z \sigma(a)+\delta(a)
$$

We consider $z$ to have degree 1 in $B$, and under this grading $B$ is a connected-graded algebra generated in degree 1 . In fact, $B:=\mathbb{T}(W) / J$ for some ideal $J$, where $W=V \oplus \mathbb{k} z$.

The following construction is a graded version of a construction by Gopalakrishnan and Sridharan in [6]. We recreate it from scratch because we will refer to the details of the construction later.

Let $V_{n} \subseteq A_{+}^{\otimes n}$ such that $A \otimes V_{\bullet}$, as a subcomplex of the bar resolution $A \otimes A_{+}^{\otimes \bullet}$, is a minimal projective resolution of the trivial module ${ }_{A} \mathbb{k}$. We may assume $V_{0}=\mathbb{k}$ and $V_{1}=V$.

Definition 2.1. We construct two chain complexes of projective $B$-modules:

(1) $P_{\bullet}:=B \otimes_{A}\left(A \otimes V_{\bullet}\right)(-1)$, which is canonically isomorphic to $B \otimes V_{\bullet}(-1)$. (Here, the degree shift is by internal degree.)

(2) $Q_{\bullet}$ is the complex with

$$
Q_{i}= \begin{cases}B \otimes V_{i}, & \text { for } i \geq 0, \\ \mathbb{k}, & \text { for } i=-1,\end{cases}
$$

and differential $\partial_{i, Q}=-\partial_{i, P}$ for $i \geq 1$, and $\partial_{0, Q}: B \otimes V^{0} \rightarrow \mathbb{k}$ given by the augmentation.

We begin by computing the homology of the complexes $P_{\bullet}$ and $Q$. 
Lemma 2.2. We have

$$
H_{i}\left(P_{\bullet}\right)= \begin{cases}\bigoplus_{n \geq 0} \mathbb{k}\left(z^{n}+B A_{+}\right)(-1), & \text { if } i=0, \\ 0, & \text { otherwise, }\end{cases}
$$

and

$$
H_{i}\left(Q_{\bullet}\right)= \begin{cases}\bigoplus_{n \geq 1} \mathbb{k}\left(z^{n}+B A_{+}\right), & \text {if } i=0, \\ 0, & \text { otherwise, }\end{cases}
$$

where $z^{n}+B A_{+} \in B /\left(B A_{+}\right)$.

Proof. Since $H_{i}\left(P_{\bullet}\right)=\operatorname{Tor}_{i}^{A}(B, \mathbb{k})(-1)$ and $B$ is a graded-free $A$-module, the first statement holds. Furthermore, $H_{i}\left(Q_{\bullet}\right)=H_{i}\left(P_{\bullet}\right)$ when $i \geq 1$. The statement about $H_{0}\left(Q_{\bullet}\right)$ and $H_{-1}\left(Q_{\bullet}\right)$ can be verified by direct calculation.

Lemma 2.3 (c.f. [6, Lemma of Theorem 1]). There exists a graded chain map $f: P_{\bullet} \rightarrow Q_{\bullet}$ with $f_{0}(b \otimes \lambda):=b z \otimes \lambda, f_{-1}:=0, \operatorname{im} f_{0} \subseteq B_{+} \otimes V_{0}$, and im $f_{1} \subseteq B_{+} \otimes V_{1}$.

Proof. By construction, im $f_{0} \subseteq B_{+} \otimes V_{0}$. We may lift $\delta: A_{1}=V_{1} \rightarrow A_{2}$ to a map $\tilde{\delta}: V_{1} \rightarrow$ $A_{1} \otimes V_{1} \subseteq B_{+} \otimes V_{1}$. Then for $x \in V_{1}$, put

$$
f_{1}(1 \otimes x):=z \otimes \sigma^{-1}(x)-\tilde{\delta}(x) \in B_{+} \otimes V_{1} .
$$

Computation shows that $\partial_{Q} \circ f_{1}=\partial_{P} \circ f_{0}$.

By Lemma 2.2, $Q$. is exact at every term except at $Q_{0}$. Therefore, the construction of the rest of the chain map follows automatically.

We can now construct a projective resolution of ${ }_{B} \mathbb{k}$ from the resolution $A \otimes V_{\bullet}$.

Lemma 2.4 (c.f. [6, Theorem 1]). The algebraic mapping cone of $f$,

$$
\begin{array}{r}
\cdots \rightarrow B \otimes\left(V_{n} \oplus V_{n-1}(-1)\right) \stackrel{\left(\begin{array}{ll}
\partial_{n} & 0 \\
f_{n} & \partial_{n-1}
\end{array}\right)}{\longrightarrow} B \otimes\left(V_{n-1} \oplus V_{n-2}(-1)\right) \rightarrow \cdots \\
\rightarrow B \otimes\left(V_{1} \oplus V_{0}(-1)\right) \stackrel{\left(\begin{array}{l}
\partial_{1} \\
f_{0}
\end{array}\right)}{\longrightarrow} B \rightarrow \mathbb{k} \rightarrow 0,
\end{array}
$$

is exact. (Here, the functions in each matrix act on the right.) Hence, if we write cone $(f)$ in the form $B \otimes W_{\bullet} \rightarrow \mathbb{k} \rightarrow 0$, then $B \otimes W_{\bullet}$ is a projective resolution of ${ }_{B} \mathbb{k}$.

Proof. We consider $f_{\bullet}: H_{\bullet}\left(P_{\bullet}\right) \rightarrow H_{\bullet}\left(Q_{\bullet}\right)$, the induced map on homology. Direct calculation shows $f_{0}: H_{0}\left(P_{\bullet}\right) \rightarrow H_{0}\left(Q_{\bullet}\right)$ is an isomorphism. By Lemma 2.2, $f_{n}: H_{n}\left(P_{\bullet}\right) \rightarrow H_{n}\left(Q_{\bullet}\right)$ is an isomorphism for $n \neq 0$. Therefore, the chain map $f$ is a quasi-isomorphism, and the algebraic mapping cone is exact (see, for example, [10, Corollary 1.5.4]).

It is not true that $B \otimes W_{\bullet} \rightarrow \mathbb{k} \rightarrow 0$ must be a minimal projective resolution. For the resolution to be minimal, $f_{n}\left(B \otimes V_{n}(-1)\right)$ must lie entirely inside $B_{+} \otimes V_{n}$ (or, equivalently, each entry of the matrix representation of each $f_{n}$ must be an element of $B_{+}$). This is not the case in Example 5.2 . 


\section{Maps between E $(A)$ And $\mathrm{E}(B)$}

In this section, we consider maps relating $\mathrm{E}(A)$ and $\mathrm{E}(B)$. Because $B \otimes W_{n}=B \otimes V_{n} \oplus B \otimes$ $V_{n-1}(-1)$, it is tempting to view $\mathrm{E}^{n}(B)$ as $\mathrm{E}^{n}(A) \oplus \mathrm{E}^{n-1}(A)(-1)$. However, there are many obstructions to this, including the fact that $\mathrm{E}(A)$ is not a subalgebra of $\mathrm{E}(B)$ and that the resolution $B \otimes W_{\bullet}$ is not minimal.

However, enough of $B \otimes W_{\bullet}$ is minimal to prove the following:

Lemma 3.1. $\mathrm{E}(\iota)\left(\mathrm{E}^{1}(B)\right)=\mathrm{E}^{1}(A)$ and $\mathrm{E}(\iota)\left(\mathrm{E}^{2}(B)\right)=\mathrm{E}^{2}(A)$, where $\iota: A \hookrightarrow B$ is the inclusion.

Proof. Since $\operatorname{im} f_{0} \subseteq B_{+} \otimes V_{0}$ and $\operatorname{im} f_{1} \subseteq B_{+} \otimes V_{1}$, the sequence

$$
B \otimes\left(V_{2} \oplus V_{1}(-1)\right) \stackrel{\left(\begin{array}{ll}
\partial_{2} & 0 \\
f_{1} & \partial_{1}
\end{array}\right)}{\longrightarrow} B \otimes\left(V_{1} \oplus V_{0}(-1)\right) \stackrel{\left(\begin{array}{l}
\partial_{1} \\
f_{0}
\end{array}\right)}{\longrightarrow} B
$$

may be extended to a minimal projective resolution $B \otimes U_{\bullet}$ of ${ }_{B} \mathbb{k}$. Now, the inclusion $\iota: A \hookrightarrow B$ induces a chain map $\tilde{\imath}: A \otimes V_{\bullet} \rightarrow B \otimes U_{\bullet}$ where $\tilde{\iota}_{1}(a \otimes x)=\iota(a) \otimes(x, 0)$ and $\tilde{\iota}_{2}(a \otimes x)=$ $\iota(a) \otimes(x, 0)$.

We now define some chain maps for use later. Let

$$
\tilde{\varphi}_{n}: \operatorname{Hom}_{\mathbb{k}}\left(V_{n-1}(-1), \mathbb{k}\right) \rightarrow \operatorname{Hom}_{\mathbb{k}}\left(V_{n} \oplus V_{n-1}(-1), \mathbb{k}\right)
$$

be the map dual to the projection $V_{n} \oplus V_{n-1}(-1) \rightarrow V_{n-1}(-1)$ onto the second direct summand. Let

$$
\tilde{\tau}_{n}: \operatorname{Hom}_{\mathbb{k}}\left(V_{n} \oplus V_{n-1}(-1), \mathbb{k}\right) \rightarrow \operatorname{Hom}_{\mathbb{k}}\left(V_{n}, \mathbb{k}\right)
$$

be the restriction to the first direct summand.

Lemma 3.2. (1) $\tilde{\varphi}_{n}$ and $\tilde{\tau}_{n}$ induce maps $\varphi_{n}: \mathrm{E}^{n-1}(A)(1) \rightarrow \mathrm{E}^{n}(B)$ and $\tau_{n}: \mathrm{E}^{n}(B) \rightarrow \mathrm{E}^{n}(A)$, respectively.

(2) $\tau_{n}=\mathrm{E}^{n}(\iota)$, where $\iota: A \hookrightarrow B$ is the inclusion.

(3) $\varphi_{n}\left(\mathrm{E}^{n-1}(A)\right)=\operatorname{ker}\left(\tau_{n}\right)$.

Proof. Recall that we have natural isomorphisms

$$
\operatorname{Hom}_{A}(A \otimes-, \mathbb{k}) \simeq \operatorname{Hom}_{\mathbb{k}}(-, \mathbb{k}) \text { and } \operatorname{Hom}_{B}(B \otimes-, \mathbb{k}) \simeq \operatorname{Hom}_{\mathbb{k}}(-, \mathbb{k}) .
$$

The map $\tilde{\varphi}_{n}$ induces a map $\varphi_{n}: \mathrm{E}^{n-1}(A) \rightarrow \mathrm{E}^{n}(B)$ because the diagram

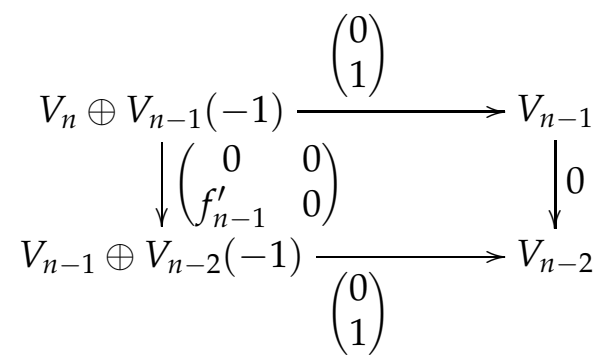

commutes, where $f_{n-1}^{\prime}$ is the composition

$$
V_{n-1}(-1) \hookrightarrow B \otimes V_{n-1}(-1) \stackrel{f_{n-1}}{\longrightarrow} B \otimes V_{n-1} \rightarrow B / B_{+} \otimes V_{n-1} \simeq V_{n-1} .
$$

We have $\tau_{n}=\mathrm{E}^{n}(\iota)$ because $\tilde{\tau}_{n}$ is dual to the chain map

$$
A \otimes V_{n} \stackrel{\iota \otimes \mathrm{id}}{\longrightarrow} B \otimes V_{n} \hookrightarrow B \otimes\left(V_{n} \oplus V_{n-1}(-1)\right) .
$$


It is clear that $\varphi_{n}(\mathrm{E}(A)) \subseteq \operatorname{ker}\left(\tau_{n}\right)$. Now, suppose $\xi \in \operatorname{Hom}_{\mathbb{k}}\left(V_{n} \oplus V_{n-1}(-1), \mathbb{k}\right)$ represents a cohomology class $[\xi] \in \operatorname{ker}\left(\tau_{n}\right) \subseteq \mathrm{E}^{n}(B)$. As $A \otimes V_{\bullet}$ is a minimal projective resolution for $A \mathbb{k}$, this implies that $\tilde{\tau}_{n}(\xi)=0$, that is, $\left.\xi\right|_{V_{n} \oplus 0}=0$. So, there exists $\xi^{\prime} \in \operatorname{Hom}_{\mathbb{k}}\left(V_{n-1}(-1), \mathbb{k}\right)$ such that $\xi=\left(0, \xi^{\prime}\right)$, that is, $\tilde{\varphi}\left(\xi^{\prime}\right)=\xi$.

Let $\bar{z} \in W^{*}$ such that $\bar{z}(z)=1$ and $\bar{z}(V)=0$. We also use the notation $\bar{z}$ to mean the induced element of bidegree $(1,1)$ in the Yoneda algebra $\mathrm{E}(B)$. Let $\mathrm{D}(A)$ be the subalgebra of $\mathrm{E}(A)$ generated as a $\mathbb{k}$-algebra by $\mathrm{E}^{1}(A)$ and $\mathrm{E}^{2}(A)$. We define $\mathrm{D}(B)$ analogously.

In the case where $A$ is $\mathcal{K}_{2}$, [3] exhibits short exact sequences

$$
0 \rightarrow \bar{z} \mathrm{E}^{n-1}(B) \rightarrow \mathrm{E}^{n}(B) \rightarrow \mathrm{E}^{n}(A) \rightarrow 0 .
$$

Our goal is to show that if $B$ is $\mathcal{K}_{2}$, there are similar short exact sequences

$$
0 \rightarrow \bar{z} \mathrm{E}^{n-1}(B) \rightarrow \mathrm{E}^{n}(B) \rightarrow \mathrm{D}^{n}(A) \rightarrow 0 .
$$

We recall some more results from [3]. As before, $\iota: A \hookrightarrow B$ is the inclusion. Noting that $A_{+} B=B A_{+}$is an ideal of $B$, set $C:=B / A_{+} B$. As a $\mathbb{k}$-algebra, $C \simeq \mathbb{k}[z]$, and has a $B$-module endomorphism $\zeta: C \rightarrow C$ via right-multiplication by $z$. Furthermore, ${ }_{B} C=B \otimes_{A} \mathbb{k}$, meaning $\operatorname{Ext}_{B}(C, \mathbb{k})=\mathrm{E}(A)$. Hence, the short exact sequence

$$
0 \rightarrow C(-1) \stackrel{\zeta}{\rightarrow} C \rightarrow \mathbb{k} \rightarrow 0
$$

yields a long exact sequence

$$
\cdots \rightarrow \mathrm{E}^{n-1}(A)(1) \stackrel{\alpha}{\rightarrow} \mathrm{E}^{n}(B) \stackrel{\mathrm{E}(\iota)}{\rightarrow} \mathrm{E}^{n}(A) \stackrel{\zeta^{*}}{\rightarrow} \mathrm{E}^{n}(A)(1) \rightarrow \cdots .
$$

(It is this sequence that breaks into the short exact sequences (1) above when $A$ is $\mathcal{K}_{2}$.) The map $\zeta^{*}: \mathrm{E}(A) \rightarrow \mathrm{E}(A)(0,1)$ is a $\mathrm{E}(\iota)$-derivation, and vanishes on $\mathrm{E}^{1}(A)$ and $\mathrm{E}^{2}(A)$.

Lemma 3.3. $\mathrm{E}(\iota)(\mathrm{D}(B))=D(A)$.

Proof. By Lemma 3.1, $\mathrm{E}(\iota)\left(\mathrm{D}^{1}(B)\right)=\mathrm{E}^{1}(A)=\mathrm{D}^{1}(A)$ and $\mathrm{E}(\iota)\left(\mathrm{D}^{2}(B)\right)=\mathrm{E}^{2}(A)=\mathrm{D}^{2}(A)$.

Lemma 3.4. Suppose $B$ is $\mathcal{K}_{2}$. Then the long exact sequence (2) breaks into short exact sequences

$$
0 \rightarrow \bar{z} \mathrm{E}^{n-1}(B) \rightarrow \mathrm{E}^{n}(B) \rightarrow \mathrm{D}^{n}(A) \rightarrow 0 \quad(n \geq 1) .
$$

Proof. As $\zeta^{*}$ is a $\mathrm{E}(\iota)$-derivation which vanishes on $\mathrm{E}^{1}(A)$ and $\mathrm{E}^{2}(A)$, and $\mathrm{D}(A)$ is generated by $\mathrm{E}^{1}(A)$ and $\mathrm{E}^{2}(A), \zeta^{*}$ vanishes on all of $\mathrm{D}(A)$. Thus, $\alpha$ in injective on $\mathrm{D}(A)(1)$. Therefore, for each $n$, we have short exact sequences

$$
0 \rightarrow \mathrm{D}^{n-1}(A)(1) \stackrel{\alpha}{\rightarrow} \mathrm{E}^{n}(B) \stackrel{\mathrm{E}(\iota)}{\rightarrow} \mathrm{D}^{n}(A) \rightarrow 0
$$

However, since $\operatorname{ker}\left(\mathrm{E}(\iota): \mathrm{E}^{1}(B) \rightarrow \mathrm{D}^{1}(A)\right)=\mathbb{k} \bar{z}$, surjectivity of $\mathrm{E}(\iota)$ implies that

$$
\alpha\left(\mathrm{D}^{n-1}(A)(1)\right)=\bar{z} \mathrm{E}^{n-1}(B) .
$$

It is no coincidence that the previous proof is very similar to the proof of [3. Theorem 10.2], in which the short exact sequence (1) appeared. Later, we will show that $B$ being $\mathcal{K}_{2}$ will imply that $A$ is also $\mathcal{K}_{2}$, meaning that the short exact sequences (1) and (3) are indeed the same.

We now relate the maps $\alpha$ and $\varphi$.

Lemma 3.5. Suppose $B$ is $\mathcal{K}_{2}$, and $\mathrm{E}^{n, m}(A)=\mathrm{D}^{n, m}(A)$. Then $\varphi_{n}$ is injective on $\mathrm{E}^{n, m}(A)(1)$.

Proof. By Lemmas 3.2 and 3.4 $\varphi\left(\mathrm{E}^{n, m}(A)\right)=\bar{z} \mathrm{E}^{n, m}(B)$. On the other hand,

$$
\operatorname{dim} \mathrm{E}^{n, m}(A)=\operatorname{dim} \mathrm{D}^{n, m}(A)=\operatorname{dim} \bar{z} \mathrm{E}^{n, m}(B),
$$

by injectivity of $\alpha$ on $\mathrm{D}^{n}(A)$. 


\section{PROOF OF THE MAIN THEOREM}

Again, we continue to use the notation established in the previous section. We are now ready to prove Theorem 1.2, which we restate.

Theorem 4.1. If $B$ is $\mathcal{K}_{2}$, then $A$ is also $\mathcal{K}_{2}$.

Proof. Suppose $A$ is not $\mathcal{K}_{2}$. Then there is a unique bidegree $(n, m)$ such that $\mathrm{E}^{n, m}(A) \neq \mathrm{D}^{n, m}(A)$ but $\mathrm{E}^{i, j}(A)=\mathrm{D}^{i, j}(A)$ when $i<n$ or when $i=n$ and $j<m$. Let $\xi: V_{n, m} \rightarrow \mathbb{k}$ represent a cohomology class $[\xi] \in \mathrm{E}^{n, m}(A) \backslash \mathrm{D}^{n, m}(A)$. Under the natural isomorphism

$$
\operatorname{Hom}_{\mathbb{k}}\left(V_{n}, \mathbb{k}\right) \simeq \operatorname{Hom}_{A}\left(A \otimes V_{n}, \mathbb{k}\right),
$$

we can also view $\xi: A \otimes V_{n} \rightarrow \mathbb{k}$.

Recall the chain map $f$ and the projective resolution $B \otimes W_{\bullet} \rightarrow \mathbb{k} \rightarrow 0$ in the previous section. Let $\xi^{\prime}$ be the map $(\xi, 0): B \otimes\left(V_{n} \oplus V_{n-1}(-1)\right) \rightarrow \mathbb{k}$.

We wish to show $\xi^{\prime}$ represents a cohomology class $\left[\xi^{\prime}\right]$ in $\mathrm{E}^{n}(B)$. Under the natural isomorphism

$$
\operatorname{Hom}_{\mathbb{k}}\left(V_{n} \oplus V_{n-1}, \mathbb{k}\right) \simeq \operatorname{Hom}_{B}\left(B \otimes\left(V_{n} \oplus V_{n-1}\right), \mathbb{k}\right),
$$

we have

$$
\partial_{B \otimes W_{\bullet}}^{*}\left(\xi^{\prime}\right)=\left(\partial_{n}^{*}(\xi), f_{n}^{*}(\xi)\right): V_{n+1, m} \oplus V_{n}(-1)_{m} \rightarrow \mathbb{k} .
$$

By degree considerations, $\partial_{n}^{*}(\xi)=0$, and therefore we need only consider $f_{n}^{*}(\xi): V_{n}(-1)_{m} \rightarrow$ $\mathbb{k}$.

Note that $V_{n}(-1)_{m}=V_{n, m-1}$. By choice of $n$ and $m, E^{n, m-1}(A)=\mathrm{D}^{n, m-1}(A)$, and so by Lemma 3.5, $\varphi: \mathrm{E}^{n, m-1}(A) \rightarrow \mathrm{E}^{n+1, m}(B)$ is injective. Therefore, any element of

$$
\operatorname{Hom}_{\mathbb{k}}\left(V_{n}(-1)_{m}, \mathbb{k}\right) \subset \operatorname{Hom}_{\mathbb{k}}\left(V_{n+1, m} \oplus V_{n}(-1)_{m}, \mathbb{k}\right)
$$

represents a nonzero cohomology class in $\mathrm{E}^{n+1, m}(B)$. Therefore, $\operatorname{Hom}_{\mathbb{k}}\left(V_{n}(-1)_{m}, \mathbb{k}\right) \cap \partial_{B \otimes W_{\bullet}}^{*}=$ 0 . Hence, $\partial_{B \otimes W_{\bullet}}^{*}\left(\xi^{\prime}\right)=0$, and $\left[\xi^{\prime}\right]$ is a cohomology class in $\mathrm{E}^{n}(B)$.

However, by Lemma 3.2, $\mathrm{E}(\iota)\left(\left[\xi^{\prime}\right]\right)=\left[\tilde{\tau}\left(\xi^{\prime}\right)\right]=[\xi]$, contrary to $\mathrm{E}(\iota)(\mathrm{E}(B))=\mathrm{D}(A)$.

\section{EXAMPLES}

We conclude with some examples. Throughout, $A\left(d_{1}, d_{2}, d_{3}, \ldots\right)$ denotes the graded-free $A$ module $A\left(d_{1}\right) \oplus A\left(d_{2}\right) \oplus A\left(d_{3}\right) \oplus \cdots$. Let $\mathrm{T}(A):=\operatorname{Tor}^{A}(\mathbb{k}, \mathbb{k})$, which is a bigraded coalgebra, with comultiplications $\mathrm{T}_{i+j}(A) \rightarrow \mathrm{T}_{i}(A) \otimes \mathrm{T}_{j}(A)$ induced by $A_{+}^{\otimes i+j} \rightarrow A_{+}^{\otimes i} \otimes A_{+}^{\otimes j}$. An algebra $A$ is $\mathcal{K}_{2}$ if and only if

$$
\Delta: \mathrm{T}^{n}(A) \rightarrow \mathrm{T}^{2}(A) \otimes \mathrm{T}^{n-2}(A) \oplus \mathrm{T}^{1}(A) \otimes \mathrm{T}^{n-1}(A)
$$

is injective.

Example 5.1. This example illustrates Theorem 1.2 by showing how some of the cohomology classes in $\mathrm{E}(A) \backslash \mathrm{D}(A)$ also appear in $\mathrm{E}(B)$. Let $A:=\mathbb{k}\langle x, y\rangle /\left\langle x^{2} y, y^{2} x\right\rangle$. Define an automorphism $\sigma: A \rightarrow A$ via $\sigma(x):=y$ and $\sigma(y):=x$. Define a $\sigma$-derivation $\delta: A(-1) \rightarrow A$ via $\delta(x)=x y$ and $\delta(y)=y x$. Let $B:=A[z ; \sigma, \delta]$ be the associated Ore extension.

A minimal projective resolution of $A_{A} \mathbb{k}$ begins

$$
\cdots \rightarrow A(-7)^{2} \stackrel{M_{4}}{\longrightarrow} A(-5)^{2} \stackrel{M_{3}}{\longrightarrow} A(-3)^{2} \stackrel{M_{2}}{\longrightarrow} A(-1)^{2} \stackrel{M_{1}}{\longrightarrow} A \rightarrow \mathbb{k} \rightarrow 0,
$$

where

$$
M_{4}:=\left(\begin{array}{cc}
y^{2} & 0 \\
0 & x^{2}
\end{array}\right), M_{3}:=\left(\begin{array}{cc}
x^{2} & 0 \\
0 & y^{2}
\end{array}\right), M_{2}:=\left(\begin{array}{cc}
y^{2} & 0 \\
0 & x^{2}
\end{array}\right), \text { and } M_{1}:=\left(\begin{array}{l}
x \\
y
\end{array}\right)
$$



but

The algebra $A$ is not $\mathcal{K}_{2}$ because $y^{2} \otimes x^{2} \otimes y$ represents a nonzero homology class in $\mathrm{T}_{3}(A)$,

$$
\begin{aligned}
\Delta\left(y^{2} \otimes x^{2} \otimes y+i m \partial\right)= & \left(y^{2} \otimes x^{2}+i m \partial\right) \otimes(y+i m \partial)+\left(y^{2}+\operatorname{im} \partial\right) \otimes\left(x^{2} \otimes y+i m \partial\right)=0 .
\end{aligned}
$$

Now, we can begin a minimal projective resolution of ${ }_{B} \mathbb{K}$ with

$$
\begin{aligned}
\ldots \rightarrow B(-7)^{2} \oplus B(-6)^{2} & \stackrel{\left(\begin{array}{cc}
M_{4} & 0 \\
f_{3} & M_{3}
\end{array}\right)}{\longrightarrow} B(-5)^{2} \oplus B(-4)^{2} \stackrel{\left(\begin{array}{cc}
M_{3} & 0 \\
f_{2} & M_{2}
\end{array}\right)}{\longrightarrow} \\
B(-3)^{2} \oplus B(-2)^{2} & \stackrel{\left(\begin{array}{cc}
M_{2} & 0 \\
f_{1} & M_{1}
\end{array}\right)}{\longrightarrow} B(-1)^{2} \oplus B(-1) \stackrel{\left(\begin{array}{c}
M_{1} \\
f_{0}
\end{array}\right)}{\longrightarrow} B \rightarrow \mathbb{k} \rightarrow 0,
\end{aligned}
$$

where

$$
\begin{array}{r}
f_{3}:=\left(\begin{array}{cc}
0 & -z-x \\
-z-y & 0
\end{array}\right) f_{2}:=\left(\begin{array}{cc}
0 & -z-y \\
-z-x & 0
\end{array}\right) \\
f_{1}:=\left(\begin{array}{cc}
0 & -z-x \\
-z-y & 0
\end{array}\right), \text { and } f_{0}:=(z) .
\end{array}
$$

Again, $y^{2} \otimes x^{2} \otimes y$ represents a nonzero homology class in $\mathrm{T}_{3}(B)$, and $\Delta\left(y^{2} \otimes x^{2} \otimes y+\operatorname{im} \partial\right)=0$, meaning $B$ is not $\mathcal{K}_{2}$.

Example 5.2. This example shows that the resolution $B \otimes W_{\bullet}$ need not be a minimal projective resolution. Let $A:=\mathbb{k}\langle w, x, y, u\rangle /\langle y u, u x-x u, u w\rangle$. The following is a minimal projective resolution for $A \mathbb{k}$ :

$$
0 \rightarrow A(-3,-4,-5, \ldots) \stackrel{M_{3}}{\longrightarrow} A(-2)^{3} \stackrel{M_{2}}{\longrightarrow} A(-1)^{4} \stackrel{M_{1}}{\longrightarrow} A \rightarrow \mathbb{k} \rightarrow 0
$$

where

$$
M_{3}:=\left(\begin{array}{ccc}
y & 0 & 0 \\
y x & 0 & 0 \\
y x^{2} & 0 & 0 \\
& \vdots
\end{array}\right), M_{2}:=\left(\begin{array}{cccc}
u & 0 & 0 & 0 \\
0 & u & 0 & -x \\
0 & 0 & 0 & y
\end{array}\right), \text { and } M_{1}:=\left(\begin{array}{c}
w \\
x \\
y \\
u
\end{array}\right)
$$

(The algebra $A$ and the resolution above first appeared in [4].) Note that $A$ is quadratic but not Koszul, and therefore is not $\mathcal{K}_{2}$.

Let $\sigma=\mathrm{id}_{A}$ and define a derivation $\delta: A(-1) \rightarrow A$ via $\delta(w)=w^{2}, \delta(x)=x^{2}, \delta(y)=y^{2}$, and $\delta(u)=u^{2}$. Let $B=A[z ; \delta]$ be the associated Ore extension. The algebra $B$ is also quadratic. 
We exhibit the chain map constructed by Lemma 2.3

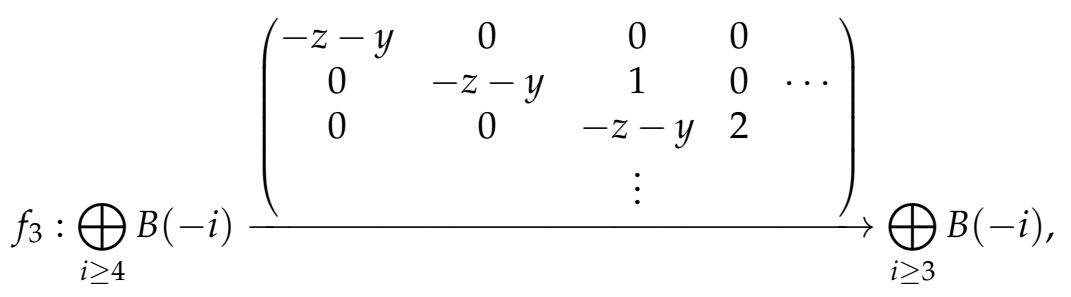

$$
\begin{aligned}
& f_{2}: B(-3)^{3} \stackrel{\left(\begin{array}{ccc}
z+u & 0 & 0 \\
0 & z+u+x & 0 \\
0 & 0 & -z-y
\end{array}\right)}{\longrightarrow} B(-2)^{3}, \\
& f_{1}: B(-2)^{4} \stackrel{\left(\begin{array}{cccc}
-z-w & 0 & 0 & 0 \\
0 & -z-x & 0 & 0 \\
0 & 0 & -z-y & 0 \\
0 & 0 & 0 & -z-u
\end{array}\right)}{\longrightarrow} B(-1)^{4} \\
& \text { and } f_{0}: B(-1) \stackrel{(z)}{\longrightarrow} B \text {. }
\end{aligned}
$$

Note the constant entries in the matrix for $f_{3}$. (To see that this is the correct matrix, use induction to show that $x^{n} z=z x^{n}+n x^{n+1}$ in $B$, and therefore $y x^{n}(z+u)=(z+y) y x^{n}+n y x^{n+1}$ in $B$.) Now, the projective resolution $B \otimes W_{\bullet}$ for ${ }_{B} \mathbb{k}$ is given by

$$
\begin{aligned}
0 \rightarrow B(-4,-5,-6, \ldots) & \stackrel{\left(\begin{array}{ll}
f_{3} & M_{3}
\end{array}\right)}{\longrightarrow} B(-3,-4,-5, \ldots,) \oplus B(-3)^{3} \frac{\left(\begin{array}{cc}
M_{3} & 0 \\
f_{2} & M_{2}
\end{array}\right)}{\longrightarrow} \\
B(-2)^{3} \oplus B(-2)^{4} & \stackrel{\left(\begin{array}{cc}
M_{2} & 0 \\
f_{1} & M_{1}
\end{array}\right)}{\longrightarrow} B(-1)^{4} \oplus B(-1) \stackrel{\left(\begin{array}{c}
M_{1} \\
f_{0}
\end{array}\right)}{\longrightarrow} B \rightarrow \mathbb{k} \rightarrow 0 .
\end{aligned}
$$

To calculate $\mathrm{E}(B)$, we apply $\operatorname{Hom}_{B}(-, \mathbb{k})$ and use the natural isomorphism $\operatorname{Hom}_{B}(B \otimes-, \mathbb{k}) \simeq$ $\operatorname{Hom}_{\mathbb{k}}(-, \mathbb{k})$ to get the sequence

$$
\begin{aligned}
0 \rightarrow \operatorname{Hom}_{\mathbb{k}}(\mathbb{k}, \mathbb{k}) \stackrel{0}{\rightarrow} \operatorname{Hom}_{\mathbb{k}}\left(\mathbb{k}(-1)^{5}, \mathbb{k}\right) \stackrel{0}{\rightarrow} \operatorname{Hom}_{\mathbb{k}}\left(\mathbb{k}(-2)^{7}, \mathbb{k}\right) \stackrel{0}{\rightarrow} \\
\quad \operatorname{Hom}_{\mathbb{k}}\left(\bigoplus_{i \geq 3} \mathbb{k}(-i) \oplus \mathbb{k}(-3)^{3}, \mathbb{k}\right) \stackrel{d}{\rightarrow} \operatorname{Hom}_{\mathbb{k}}\left(\bigoplus_{i \geq 4} \mathbb{k}(-i), \mathbb{k}\right) \rightarrow 0 .
\end{aligned}
$$

For $j \geq 3$, let $\lambda_{j} \in \operatorname{Hom}_{\mathbb{k}}\left(\oplus_{i \geq 3} \mathbb{k}(-i) \oplus \mathbb{k}(-3)^{3}, \mathbb{k}\right)$ be the map $\bigoplus_{i \geq 3} \mathbb{k}(-i) \rightarrow \mathbb{k}$ by projecting on the $(j-2)$ th coordinate. Similarly define $\rho_{j} \in \operatorname{Hom}_{\mathbb{k}}\left(\oplus_{i \geq 4} \mathbb{k}(-i), \mathbb{k}\right)$ for $j \geq 4$. Then one can show that for $j \geq 5, d\left(\lambda_{j}\right)=(j-3) \rho_{j}$, but that $d\left(\lambda_{3}\right)=0$ and $d\left(\lambda_{4}\right)=0$. Hence $\mathrm{E}^{3,4}(B) \neq 0$, meaning $B$ is not Koszul (and therefore not $\mathcal{K}_{2}$, since $B$ is quadratic).

\section{REFERENCES}

[1] Roland Berger, Koszulity for nonquadratic algebras, J. Algebra 239 (2001), no. 2, 705-734. MR1832913 (2002d:16034)

[2] Thomas Cassidy, Christopher Phan, and Brad Shelton, The Yoneda algebra of a $\mathcal{K}_{2}$ algebra need not be another $\mathcal{K}_{2}$ algebra, Comm. Algebra 38 (2010), no. 1, 46-48.

[3] Thomas Cassidy and Brad Shelton, Generalizing the notion of Koszul algebra, Math. Z. 260 (2008), no. 1, 93-114. MR2413345 (2009e:16047) 
[4] $\longrightarrow$ PBW-deformation theory and regular central extensions, J. Reine Angew. Math. 610 (2007), 1-12. MR2359848 (2008j:16090)

[5] K. R. Goodearl and R. B. Warfield Jr., An introduction to noncommutative Noetherian rings, 2nd ed., London Mathematical Society Student Texts, vol. 61, Cambridge University Press, Cambridge, 2004. MR2080008 (2005b:16001)

[6] N. S. Gopalakrishnan and R. Sridharan, Homological dimension of Ore-extensions, Pacific J. Math. 19 (1966), 67-75. MR0200324 (34 \#223)

[7] Christopher Phan, Generalized Koszul properties for augmented algebras, J. Algebra 321 (2009), no. 5, $1522-1537$. MR2494406 (2010a:16017)

[8] Alexander Polishchuk and Leonid Positselski, Quadratic algebras, University Lecture Series, vol. 37, American Mathematical Society, Providence, RI, 2005. MR2177131 (2006f:16043)

[9] Stewart B. Priddy, Koszul resolutions, Trans. Amer. Math. Soc. 152 (1970), 39-60. MR0265437 (42 \#346)

[10] Charles A. Weibel, An introduction to homological algebra, Cambridge Studies in Advanced Mathematics, vol. 38, Cambridge University Press, Cambridge, 1994. MR1269324 (95f:18001)

Department of MAthematics, University of Glasgow, Glasgow G12 8QW, United Kingdom E-mail address: c.phan@maths.gla .ac.uk

URL: http://www.maths.gla.ac.uk/〜 clphan/ 